\title{
PLAN DE GESTIÓN SUSTENTABLE DE RIESGO DE INUNDACIÓN: UNA PROPUESTA DESDE CARABOBO, VENEZUELA
}

\author{
Bettys Farias ${ }^{1, *}$, Adriana Marquez ${ }^{1}$, Edilberto Guevara ${ }^{1}$ y Demetrio Rey ${ }^{2}$
}

\section{RESUMEN}

En esta investigación aborda la formulación de un plan de gestión sustentable de riesgo de inundación durante el periodo 2015-2017 en el Municipio Naguanagua, Carabobo, Venezuela, con el objetivo de mejorar la capacidad de preparación y de respuesta ante situaciones de desastres en las comunidades afectadas por las inundaciones. Su novedad se basa en el método de integración de actores públicos y academia a diferentes niveles con comunidades locales. Esta investigación es de tipo descriptiva, el diseño es un trabajo de campo donde la información se obtiene mediante la aplicación de entrevistas, encuestas y observación directa. Las actividades de investigación se orientaron a promover estrategias y criterios educativos encaminados a la reducción del riesgo de inundación mediante la creación de capacidades en la población para superar amenazas y contribuir, de ese modo, a formar una comunidad resiliente. Desde esta experiencia se concluye que es posible desarrollar proyectos donde la alianza Universidad-Comunidad se fortalezca para lograr la sostenibilidad del desarrollo social y económico de una población, requiriendo la integración efectiva de la gestión del riesgo de desastres en sus políticas, planes de desarrollo, proyectos y acciones dirigidas al conocimiento y comportamiento de la sociedad ante la presencia de fenómenos hidrometeorológicos.

\section{PALABRAS CLAVE}

Inundación, Gestión de riesgo, Vulnerabilidad, Venezuela

\section{SUSTAINABLE FLOOD RISK MANAGEMENT PLAN: A PROPOSAL FROM CARABOBO, VENEZUELA}

\begin{abstract}
This research addresses the development of a sustainable flood risk management plan during 2015-2017 in the Municipality Naguanagua, Carabobo, Venezuela. This aims to improve the preparedness and response capacities to disaster situations in flood-affected communities. Its novelty bases on its method for integration for academia and public actors at different levels with local communities. This research is descriptive and supported by field work research where information is obtained through the application of interviews, surveys and direct observation. Research activities were aimed at promoting educational strategies and criteria aimed at reducing flood risk by building capacities in the population to overcome threats and thereby contribute to building a resilient community. From this experience, conclusions point out that it is possible to develop projects where the University-Community alliance is strengthened to achieve the sustainability of the social and economic development of a population. Nevertheless, this requires an effective integration of disaster risk management: its policies, development plans, projects and actions that look at societal knowledge and behavior in the presence of hydrometeorological phenomena.
\end{abstract}

\section{KEYWORDS}

Flooding, Risk management, Vulnerability, Venezuela
1. Centro de Investigaciones Hidrológicas y Ambientales, Universidad de Carabobo, Carabobo, Venezuela.

2. Instituto de Matemática y Computo Aplicado, Universidad de Carabobo, Carabobo, Venezuela.

*Autor de correspondencia: bettysfarias@gmail.com

\section{RECIBIDO}

30 de abril de 2019

\section{ACEPTADO}

10 de noviembre de 2019

\section{PUBLICADO}

1 de enero de 2020

\section{Formato cita}

Recomendada (APA): Farias, B., Marquez, A., Guevara, E. \& Rey, D. (2020). Plan de Gestión Sustentable de Riesgo de Inundación: Una Propuesta desde Carabobo, Venezuela. Revista de Estudios Latinoamericanos sobre Reducción del Riesgo de Desastres REDER, 4(1), pp.67-79.

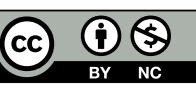

Todos los artículos publicados en REDER siguen una política de Acceso Abierto y se respaldan en una Licencia CreativeCommons Atribución-NoComercial 4.0 Internacional.

\section{Revista de Estudios}

Latinoamericanos sobre Reducción del Riesgo de Desastres (REDER)

Diseño: Lupe Bezzina Tipografia: Hospital 


\section{INTRODUCCIÓN}

Las inundaciones son una de las amenazas más frecuentes, y es un hecho ampliamente confirmado que han provocado diferentes tipos de afectaciones en las comunidades de todo el mundo. Uno de los motivos de incertidumbre no es la presencia de las inundaciones, porque ellas son parte del ciclo hidrológico y climático del planeta, sino su frecuencia y su intensidad. Sin dejar a un lado, que hay otros factores determinantes en el grado de afectaciones o daños que las inundaciones causan, como lo son las condiciones sociales de las poblaciones.

Las inundaciones no son desastres naturales sino fenómenos naturales que responden a las pautas habituales del flujo de los ríos. Mientras que los desastres son obra de los hombres en tanto que han buscado el riesgo ocupando las planicies de inundación de los ríos con viviendas, industrias, carreteras, puentes, entre otros ya sea por ignorancia o conveniencia económica (Farías et al., 2017)

Para diagnosticar sitios de posibles desbordes de cuerpos de agua y planificar acciones para mitigar la ocurrencia de inundaciones, se recomienda el uso de la tecnología de los sistemas de Información geográfica (SIG), integrando modelos hidráulicos e hidrológicos, así como las imágenes satelitales generadas desde sensores remotos (SR) que facilitan la caracterización de la distribución espacial de los asentamientos humanos dentro de las zonas urbanas y rurales (Farías et al., 2018) y la posición relativa respecto de elementos naturales (ríos, lagos, montañas, entre otros). Los productos de aplicación de SIG y SR contribuyen a la toma de decisiones en todas las etapas de cualquier Plan de Gestión de Riesgo de Inundación, ya que permiten determinar de forma precisa las planicies de inundación que han sido, desde siempre, los lugares preferidos para la actividad socioeconómica como lo demuestran las altas densidades de asentamientos humanos que allí se suelen encontrar. Según el informe anual 2016 de la Organización Meteorológica Mundial plantea que en 2015 el $97 \%$ de los desastres se debieron a riesgos asociados con el tiempo, clima o el agua, lo que dio lugar a pérdidas generales que ascendieron a 97.000 millones de dólares de los Estados Unidos, confirmando como una de las causas principales de estas pérdidas de vidas y de bienes materiales la atracción desde épocas muy remotas por las planicies de inundación; primero debido al rico suelo aluvial; luego, por la necesidad de acceso a fuentes de agua, transporte fluvial, desarrollo de energía y, más tarde como lugar relegado para la urbanización, particularmente para familias de bajos ingresos (OEA, 1993).

Ante esta situación es importante la presencia de políticas gubernamentales de planes de organización territorial y de gestión de riesgos de inundaciones eficientes y bien dirigidos, con suficiente capacidad organizativa interinstitucional, personal capacitado, entre otros, para poder llegar a los sectores más necesitados de la sociedad que, en presencia de amenazas de origen climático como las lluvias intensas, evidencian su vulnerabilidad física, económica y social. Desde esta premisa surge la necesidad de fortalecer mediante estrategias educativas la promoción de la prevención ante la inminente presencia de las amenazas, siendo de particular interés la importancia de criterios que favorecen la preparación y prevención como una estrategia fundamental de la gestión de riesgos de desastres (Farías et al., 2015), y como puede convertirse la prevención en una estrategia indispensable para el desarrollo sostenible de las comunidades (Guevara, 2003; 2008; 2010). Conocer las actitudes, las percepciones, hábitos y conductas de las comunidades, permite establecer acciones que coordinadas a través de un plan de gestión de riesgos procuren la participación, el apoyo continuo y alianzas estratégicas entre los sectores involucrados, mejoras en la calidad de vida, y la capacitación continua de las comunidades, que conlleven a prevenir y mitigar las consecuencias negativas de las inundaciones.

Desde el punto de vista de la sustentabilidad, considerar el tema de la planificación integral y la reducción de los riesgos es importante, ya que un municipio que no sea resiliente ante eventos extremos de origen natural conceptualmente no es sustentable. El modelo el desarrollo sostenible toma en cuenta las esferas social, institucional, ambiental y económica, siendo el modelo que satisface las necesidades de la población actual, sin comprometer las generaciones subsiguientes en satisfacer las suyas. Los objetivos ambientales de la reducción de riesgo persiguen el conocimiento del territorio (amenazas y vulnerabilidades), ordenación del territorio, protección del hábitat, modelos sostenibles de consumo, producción y crecimiento poblacional, estos objetivos están en concordancia con lo que promueve el desarrollo sostenible. 
Es así como desde la Universidad de Carabobo (UC) y del Centro de Investigaciones Hidrológicas y Ambientales (CIHAM-UC) se planteó aprovechar el recurso humano formado y el que se está formando para que se estimule y desarrolle una perspectiva transformadora hacia los problemas sociales, es decir proyectarse hacia el medio exterior de manera eficiente y organizada, con una orientación en la misión de educar y transformar mediante una interacción permanente y continua entre la universidad y la comunidad, de tal manera que se contribuya a mejorar las condiciones sociales y medioambientales de la comunidad. A su vez se contribuye al cumplimiento de los Objetivos de Desarrollo Sostenible (ODS) de las Naciones Unidas y el Acuerdo de París, el Marco de Sendai para la Reducción del Riesgo de Desastres 2015-2030, que tiene por objeto reducir de forma sustancial la mortalidad causada por desastres a nivel mundial para 2030 y lograr ciudades y asentamientos humanos inclusivos, seguros, resilientes y sostenibles.

En este trabajo se presenta la investigación que sustenta la formulación de un Plan de Gestión Sustentable de Riesgo de Inundación del Municipio Naguanagua (PGSRI-MN), el cual se desarrolló en seis fases: Análisis y priorización de riesgos; Caracterización del escenario de emergencia o desastre; Inventario de los recursos locales; Organización de acciones posibles de respuesta; Presentación y discusión de resultados; y Validación y Sostenibilidad del PGSRI-MN.

\section{METODOLOGIA}

\section{Área de estudio}

Esta investigación se desarrolló en el Municipio Naguanagua (MN) ubicado en la zona CentroNorte del Estado Carabobo, República Bolivariana de Venezuela. Tiene una superficie de $188 \mathrm{~km}^{2}$, representando un 4,3\% de la superficie de todo el Estado Carabobo y posee una población estimada de 157.437 habitantes según el Censo Nacional de 2011.

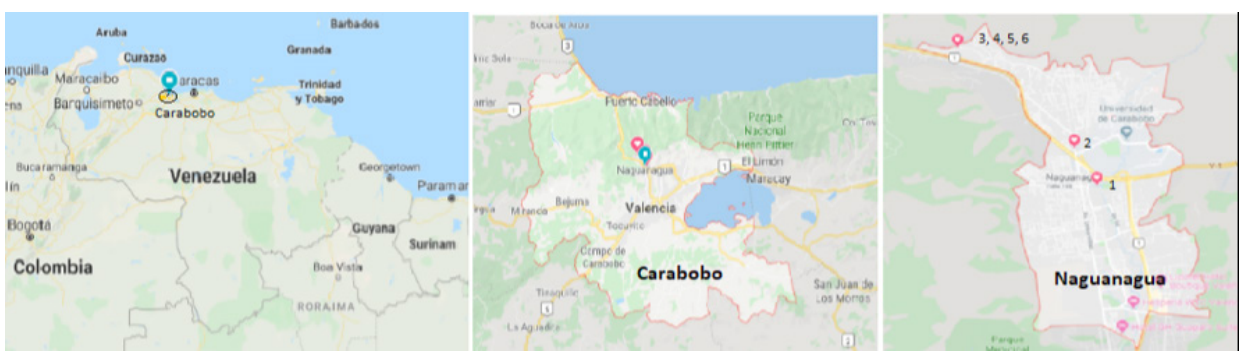

Figura 1. Ubicación geográfica del municipio Naguanagua en la región Centro-Norte del estado Carabobo de la República Bolivariana de Venezuela, y de las comunidades donde se realizó la investigación Fuente: Autores, 2020, basado en Google Maps.

EI PGRI-MN se ha implementado en seis comunidades del Municipio Naguanagua: 1) Brisas del Café, 2) Las Adjuntas, 3) Carialinda I, 4) Carialinda II, 5) Carialinda III, y 6) Montesión. Los resultados presentados corresponden a la Comunidad Popular Las Adjuntas (CPLA MN).

EI PGSRI-MN define los lineamientos básicos en materia de conocimiento del riesgo, intervención del mismo y manejo de inundaciones para el MN, así como las acciones concretas, los modelos de organización y los procedimientos generales aplicables para afrontar eficientemente una situación de desastre o emergencia previamente identificada y evaluada, con el fin de mitigar y reducir el impacto negativo sobre una comunidad como consecuencia de la ocurrencia de un evento potencialmente dañino. A continuación, se describen las fases de investigación.

\section{Análisis y priorización del riesgo}

Esta fase involucra las siguientes actividades: a) análisis general de la situación actual del Municipio Naguanagua, b) identificación del escenario según el criterio amenazante. La primera actividad involucró la caracterización biofísica de la cuenca donde está ubicada el municipio, identificación de sus cuerpos de agua, condiciones climáticas, relieve, crecimiento urbano, geología y uso del suelo, actividades económicas, infraestructura vial, situación socioeconómica, servicios públicos disponibles en el municipio. Para la identificación del escenario según el criterio amenazante se realizaron consultas comunitarias e institucionales desarrolladas mediante la aplicación de encuestas y conversatorios con los habitantes en visitas puerta a puerta a cada familia de las distintas comunidades del MN que participaron en la investigación.

\section{Caracterización general del escenario de riesgo}

En esta fase se evaluaron las siguientes actividades: a) análisis de los antecedentes del escenario de inundaciones en el Municipio en el periodo de estudio de esta investigación 
(2015-2017); b) los daños y pérdidas presentadas; c) descripción del fenómeno amenazante; d) causas el fenómeno amenazante; e) determinación de factores que favorecen la ocurrencia de las inundaciones; f) identificación de los actores involucrados en las causas del fenómeno. Estas actividades se realizaron a través de búsqueda de material bibliográfico, de revisión de antecedentes de inundaciones reportadas por Protección Civil, así como trabajo de campo donde a través de observación directa, encuestas y entrevistas se recolecto la información necesaria.

\title{
Inventario de recursos locales
}

En esta fase se realizó un inventario de recursos locales en la comunidad seleccionada. En esta etapa fue muy importante la participación de las comunidades para reconocer los recursos naturales y bienes productivos con que se cuentan y de esta forma lograr un plan de gestión de riesgos de inundación eficiente, ya que es necesario para determinar las zonas más propensas a la ocurrencia de inundaciones y /o emergencias. Se elaboraron croquis, levantamientos y mapas comunitarios donde se refleja el inventario de recursos locales y los recursos humanos con los que cuentan las comunidades tales como: centros de atención médica, iglesias, bodegas, farmacias, colegios, zonas seguras y posibles refugios. Además, se levanta la información correspondiente a ubicación de las viviendas de los líderes comunitarios, miembros de juntas comunales y otras personas importantes para el desarrollo de estas actividades.

\section{Organización de acciones posibles de respuestas}

Esta fase consiste en definir las medidas de reducción de la amenaza tanto de tipo no estructurales como medidas estructurales adecuadas a la comunidad.

Medidas no estructurales: Es cualquier medida que no suponga una construcción física y que utiliza el conocimiento, las prácticas o los acuerdos existentes para reducir el riesgo y sus impactos, especialmente a través de políticas y leyes, una mayor conciencia pública, la capacitación y la educación.

El Marco de Sendai para la Reducción del Riesgo de Desastres 2015-2030 aprobado en la Tercera Conferencia Mundial de las Naciones Unidas sobre la Reducción del Riesgo de Desastres establece entre sus prioridades: 'Comprender el riesgo de desastres', donde establece:

\begin{abstract}
"Impartir conocimientos a los funcionarios públicos a todos los niveles, la sociedad civil, las comunidades y los voluntarios, así como el sector privado, mediante el intercambio de experiencias, enseñanzas extraídas y buenas prácticas y mediante la capacitación y la educación sobre la reducción del riesgo de desastres, en particular usando los mecanismos existentes de capacitación y educación y de aprendizaje entre pares"
\end{abstract}

(UNISDR, 2015, p.19)

En esta investigación se establecieron las siguientes medidas no estructurales:1) Implementar planes de educación y divulgación sobre las acciones de gestión de riesgo de inundación, planes de ordenamiento del municipio, y manejo de cuencas; 2) Realizar mantenimiento a las obras hidráulicas de control y protección; 3) Diseñar e implementar SAT. La metodología empleada para la implementación del SAT se fundamentó en las instrucciones y recomendaciones presentadas en el Manual para docentes sobre Gestión para la Reducción del Riesgo de Desastres y Sistemas de Alerta Temprana (UNESCO, 2012).

La metodología empleada en esta fase también se fundamentó en la implementación de cursos, reuniones, charlas y talleres con los grupos de trabajo que conforman esta investigación: Universidad de Carabobo (representada por estudiantes y docentes del CIHAM-UC y Bomberos Universitarios), Protección Civil Carabobo, Protección Civil Naguanagua, y escuela Nuestra Señora de Fátima ubicada en CPLA. Se realizó además un entrenamiento por parte de Protección Civil Carabobo al personal de la UC, requisito necesario para participar con ellos en los trabajos de campo, este curso de duración 4 semanas incluyó actividades de primeros auxilios, de salvamento, de conceptos básicos de gestión de riesgo, evaluación de situaciones de emergencias, simulacros de desastres, entre otros temas.

En esta fase se ejecutó un programa denominado: Planificar Para Prevenir el Riesgo de Inundación, con el objetivo de promover estrategias orientadas a fortalecer las capacidades de actores locales, de instituciones públicas y privadas para la preparación y respuesta ante situaciones de emergencia. Este programa incluyó: un primer taller comunitario dirigido a la conformación de 
un comité comunitario de base y adiestrar sobre la importancia de los recursos ambientales para la prevención de las inundaciones; y un segundo taller que involucra acciones dirigidas a la reflexión sobre la situación de exposición al riesgo de la comunidad, ubicación de los hogares-personas más vulnerables, profundizar los conceptos de amenaza, vulnerabilidad y riesgo para la elaboración de los escenarios de riesgo, determinar las acciones necesarias para la reducción del riesgo; un tercer taller que involucra acciones dirigidas a la preparación y respuesta local, elaboración de croquis de zonificación de amenazas, exposición, vulnerabilidad y riesgo por inundación, teniendo en cuenta la ubicación del río Cabriales y sus afluentes, elaboración de croquis para identificar los recursos existentes (humanos, y materiales) en la comunidad, Identificación de zonas seguras y rutas de evacuación en caso de inundación, identificación de albergues temporales, centros de acopio y centro de operaciones de emergencia local. Todos los croquis realizados por los miembros de la comunidad fueron digitalizados por los miembros del CIHAM-UC utilizando SIG como herramientas tecnológicas que integran operaciones de gestión de bases de datos con gran potencial de análisis de información, con capacidad para visualizar información espacial como un elemento importante para comunicar, difundir e intercambiar conocimiento y proporcionan una plataforma base para interpretar cómo factores físicos, sociales y económicos interactúan en un contexto espacial (Vásquez, 2014). El Marco de Sendai promueve el uso de información espacial local, incluidos los sistemas de información geográfica (SIG), y utilizar las innovaciones en materia de tecnología de la información y las comunicaciones para mejorar los instrumentos de medición y la recopilación, el análisis y la difusión de datos. Una vez elaborados los diferentes mapas se socializaron con el resto de la comunidad. Por último se desarrolló un cuarto taller para establecer proyectos prioritarios para la reducción del riesgo, definir la metodología a aplicar para enfrentar y afrontar situaciones de riesgos de inundación y asignar responsables para el cumplimiento de las actividades, SAT integrado por pluviómetros comunitarios, linnímetro y sensores de nivel del agua en el río, conectados a un tablero de control con una alarma sonora y una visual que indican a las personas que el nivel de agua va alcanzando los umbrales definidos de acuerdo a la experiencia de los habitantes.

\section{Medidas estructurales}

Se refiere a cualquier construcción física para reducir o evitar los posibles impactos de las amenazas, o la aplicación de técnicas de ingeniería para lograr la resistencia y la resiliencia de las estructuras o de los sistemas frente a las amenazas. Este tipo de medidas nunca pueden eliminar por completo el riesgo de inundaciones. No obstante, por su presencia física, pueden dar una falsa idea de seguridad, que puede conducir al uso inadecuado de la tierra en las zonas protegidas.

Para definir la medida estructural a implementar se realizó una simulación del tramo del río Retobo donde está ubicada la CPLA, utilizando el software HEC-RAS 4.0, que es un modelo hidrológico desarrollado en el entorno SIG. Este modelo requiere características topológicas de la red hidrológica, la forma de las secciones transversales de los cauces, recorrido de los flujos de agua, coeficientes de Manning, e información de tipo espacial que se gestiona mediante los SIG. La combinación de HEC-RAS con la de los programas SIG como el HEC-GeoRAS, permite hacer la modelación de los cauces, y ser utilizados para el estudio de problemas relacionados con disponibilidad de agua, drenaje urbano, predicción de inundaciones, impacto de urbanización futura, diseño de aliviaderos para represas, reducción de daños por inundaciones, legislación sobre planicies de inundación entre otros

\section{PRESENTACIÓN Y DISCUSIÓN DE RESULTADOS \\ Análisis y priorización del riesgo}

Como resultado de esta fase se presenta la Figura 2 que muestra las áreas de la comunidad expuestas a la amenaza de inundación, producto de su ubicación dentro de la planicie inundable de los ríos Retobo, Cabriales y quebrada San Jea, demarcada en color azul.

En esta fase se dio respuestas a las siguientes interrogantes ¿Cuál es el tamaño de la población? ¿Cómo está distribuida?, ¿Cuántos hogares hay en la comunidad? ¿Qué tan a menudo la comunidad se ve afectada por desastres y amenazas?, ¿Es la incidencia cada vez mayor la comunidad? ¿Cuáles son los motivos principales de la vulnerabilidad en la comunidad? 


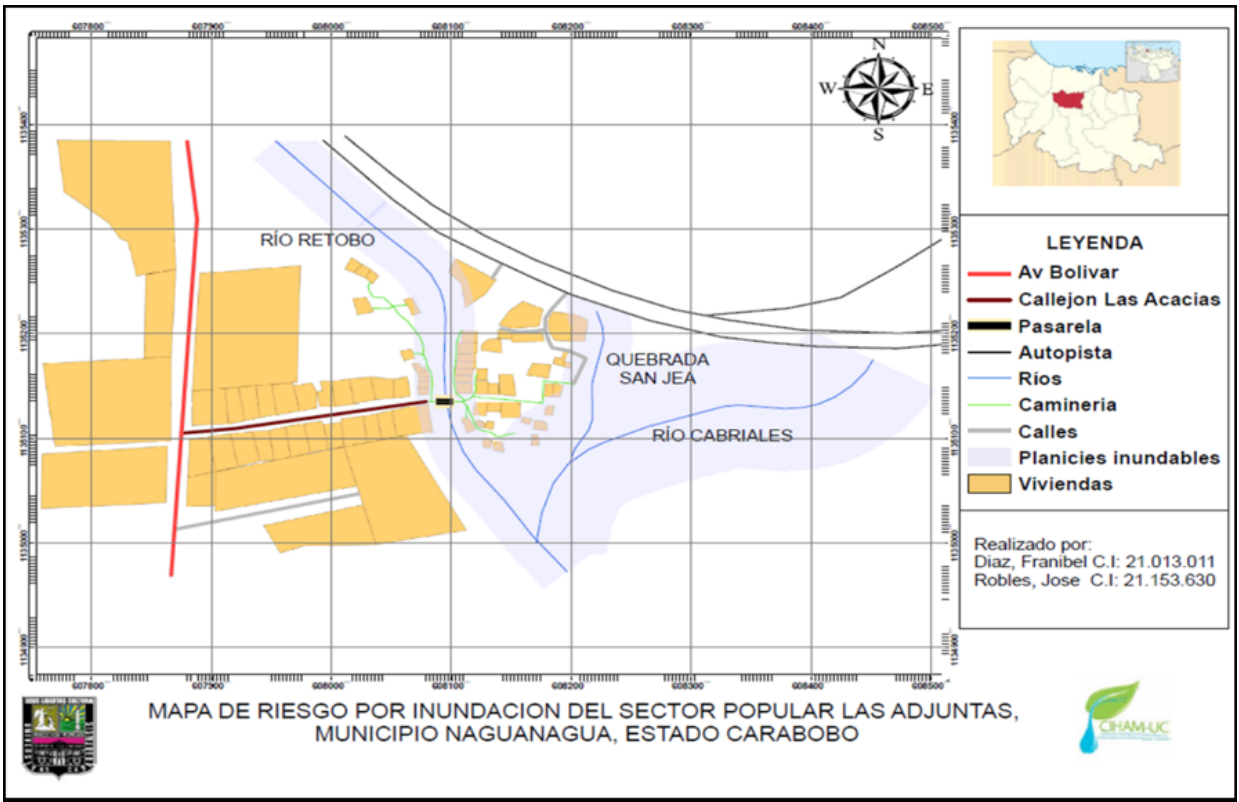

Figura 2. Áreas con exposición a la amenaza de inundación Fuente: Autores, 2020.

La Figura 3 muestra algunos de los resultados obtenidos en las encuestas realizadas a las comunidades y a los organismos que integraron el equipo de trabajo. La Figura $3 \mathrm{~A}$ representa la distribución de la población de la comunidad Las Adjuntas integrada por un total de 46 viviendas, y 368 personas, distribuida en $56 \%$ de adultos y adolescentes, $31 \%$ de niños, $9 \%$ ancianos y $4 \%$ de personas con discapacidad. La Figura 3B muestra la distribución de los eventos que han ocurrido con más frecuencia en esta comunidad, donde se observa que el $83 \%$ de la población coincide que las inundaciones son el fenómeno natural que ha ocurrido con más frecuencia en la comunidad, mientras que el otro $17 \%$ opina que han sido los vientos fuertes descritos por ellos como huracanados. Por último, la Figura $3 \mathrm{C}$ muestra la respuesta a la pregunta si ellos creen que viven en situación de riesgos, el $87 \%$ reconoce vivir expuesto a riesgos de inundación.

A

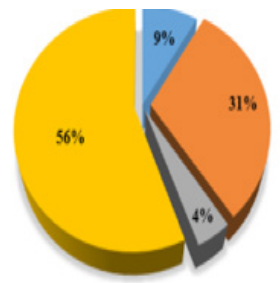

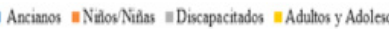
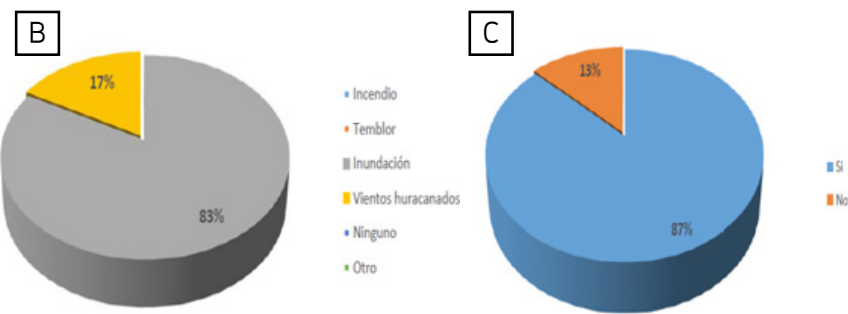

Figura 3. Resultados de encuestas aplicadas a los miembros de la comunidad las Adjuntas Fuente: Autores, 2020.

De las visitas de campo realizadas para el diagnóstico se puede observar en la Figura 4 diferentes escenas de la situación actual de exposición y vulnerabilidad de la comunidad Las Adjuntas, como por ejemplo la ubicación de las viviendas dentro de las planicies de inundación del río Retobo.
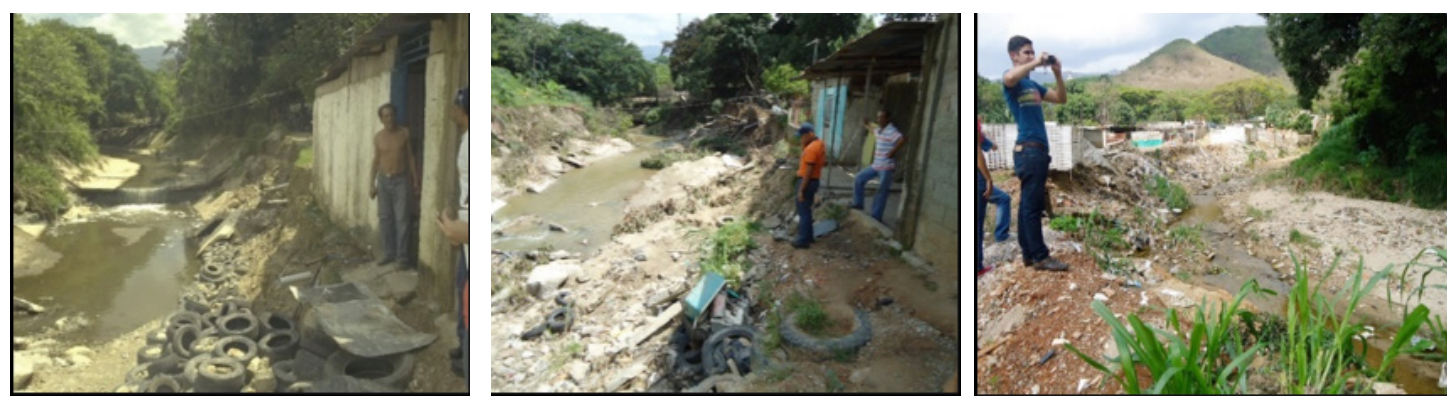

Figura 4. Escenas de ubicación de las viviendas de comunidad Las Adjuntas Fuente: Autores, 2020. 
En la Tabla 1 se presentan las diferentes vulnerabilidades o susceptibilidades ambiental, física, social, institucional, y económica que tiene la comunidad expuesta a inundaciones.

\begin{tabular}{ll}
\hline Vulnerabilidad ambiental & $\begin{array}{l}\text { El río Retobo que forma parte de la comunidad presenta exceso de malezas, } \\
\text { erosión del suelo y grandes cantidades de basura que contribuyen a su } \\
\text { contaminación influyendo negativamente en el desarrollo de sus actividades. }\end{array}$ \\
\hline Vulnerabilidad física & $\begin{array}{l}\text { Las infraestructuras más vulnerables son las viviendas construidas con materia } \\
\text { de desecho, ubicadas dentro de las planicies de inundación, ausencia de drenajes } \\
\text { y de servicios públicos. La falta de vías de comunicación con el resto de las } \\
\text { comunidades, solo tienen una pasarela que hace más vulnerable al momento de } \\
\text { desalojar ante una inundación. }\end{array}$ \\
\hline Vulnerabilidad social & $\begin{array}{l}\text { Existe poca o ninguna organización comunitaria que les permita estar bien } \\
\text { preparados para el manejo del riesgo, la mitigación y la prevención ante los } \\
\text { desastres asociados a las inundaciones. }\end{array}$ \\
\hline Vulnerabilidad institucional & $\begin{array}{l}\text { Actualmente la comunidad no dispone de apoyo por parte de los organismos } \\
\text { encargados de brindar tanto apoyo institucional como técnico, poseen } \\
\text { instrumentos para efectuar la medición de lluvia, monitoreo y alerta de los } \\
\text { niveles de agua en el rí, pero se requiere de programas de capacitación } \\
\text { permanentes en materia de sistemas de alerta temprana, que deben ser } \\
\text { apoyados por Alcaldía y otros entes gubernamentales. }\end{array}$ \\
\hline Vulnerabilidad económica & $\begin{array}{l}\text { Los habitantes de la comunidad son personas de escasos recursos económicos } \\
\text { lo cual influye directamente en la capacidad de respuesta y recuperación ante } \\
\text { situaciones de desastres. }\end{array}$ \\
\hline
\end{tabular}

Tabla 1. Tipo de vulnerabilidades en la comunidad Las Adjuntas

Fuente: Autores, 2020.

\section{Caracterización general del escenario de riesgo}

En esta comunidad existe una amenaza o peligro latente de que un evento de origen natural, como las lluvias causantes de las inundaciones, se presente con una severidad suficiente para causar pérdidas de vidas, lesiones u otros impactos en la salud, así como también daños y pérdidas en los bienes, la infraestructura, los medios de sustento, la prestación de servicios y los recursos ambientales.

La Tabla 2 presenta los resultados de la descripción del escenario de riesgo por inundación.

\begin{tabular}{|c|c|}
\hline $\begin{array}{l}\text { Antecedentes escenario } \\
\text { del riesgo, daños y pérdidas } \\
\text { presentados }\end{array}$ & $\begin{array}{l}\text { "En el mes de agosto de } 2015 \text {, producto de las fuertes lluvias en el sector popular } \\
\text { Las Adjuntas } 20 \text { viviendas fueron prácticamente devastadas este desastre } \\
\text { fue atendido por representantes de Protección civil y colaboradores de todo el } \\
\text { Estado." (J. Vásquez, entrevista personal, enero 27,2018) } \\
\text { "La CPLA-MN se encuentra en una vulnerabilidad latente debido a su cercanía al } \\
\text { río Retobo, en el mes de abril del } 2016 \text { donde una crecida causada por una lluvia } \\
\text { intensa ocasionó la socavación de las orillas del río, dejando cuatro hogares con } \\
\text { posibilidad de caer al vacío y aproximadamente a } 30 \text { familias afectadas, como } \\
\text { consecuencia de la construcción de viviendas en zonas con alto riesgo." } \\
\text { (J. Velásquez, entrevista personal, Marzo15, 2018). }\end{array}$ \\
\hline $\begin{array}{l}\text { Descripción del fenómeno } \\
\text { amenazante }\end{array}$ & $\begin{array}{l}\text { La CPLA-MN presenta riesgos considerables por inundaciones, está asentada en } \\
\text { un terreno que limita con los ríos El Retobo y Cabriales, siendo el primero el que } \\
\text { causa mayor impacto a la comunidad. }\end{array}$ \\
\hline $\begin{array}{l}\text { Identificación de causas del } \\
\text { fenómeno amenazante }\end{array}$ & $\begin{array}{l}\text { El cambio climático destrucción de la capa vegetal por factores antrópicos o } \\
\text { naturales (sequía, incendios), disminución de la infiltración, incremento de la } \\
\text { escorrentía y erosión masiva, ocurrencia de sismos desestabilizadores de masas } \\
\text { de materiales. }\end{array}$ \\
\hline $\begin{array}{l}\text { Identificación de factores } \\
\text { que favorecen la condición } \\
\text { de amenaza }\end{array}$ & $\begin{array}{l}\text { Las pendientes, constitución de los suelos, la tectónica local y regional, así como } \\
\text { la actividad antrópica, representada en la deposición de sólidos, el mal manejo de } \\
\text { aguas, canalizaciones de aguas, rellenos, sistema vial deficiente. }\end{array}$ \\
\hline $\begin{array}{l}\text { Identificación de actores } \\
\text { significativos en la condición } \\
\text { de amenaza }\end{array}$ & $\begin{array}{l}\text { Las autoridades locales responsables de la protección del medio ambiente y las } \\
\text { organizaciones comunitarias y sociales. De igual forma las comunidades que } \\
\text { viven en áreas de riesgo de inundación. }\end{array}$ \\
\hline
\end{tabular}

Tabla 2. Descripción del escenario de riesgo por inundación

Fuente: Autores, 2020. 


\section{Inventario de recursos locales}

Una de las actividades ejecutadas en esta fase fue la construcción del mapa de organización comunitaria que refleja recursos comunitarios como la casa de reuniones, la ubicación de las viviendas de los líderes comunitarios, mostrados en la Figura 5.

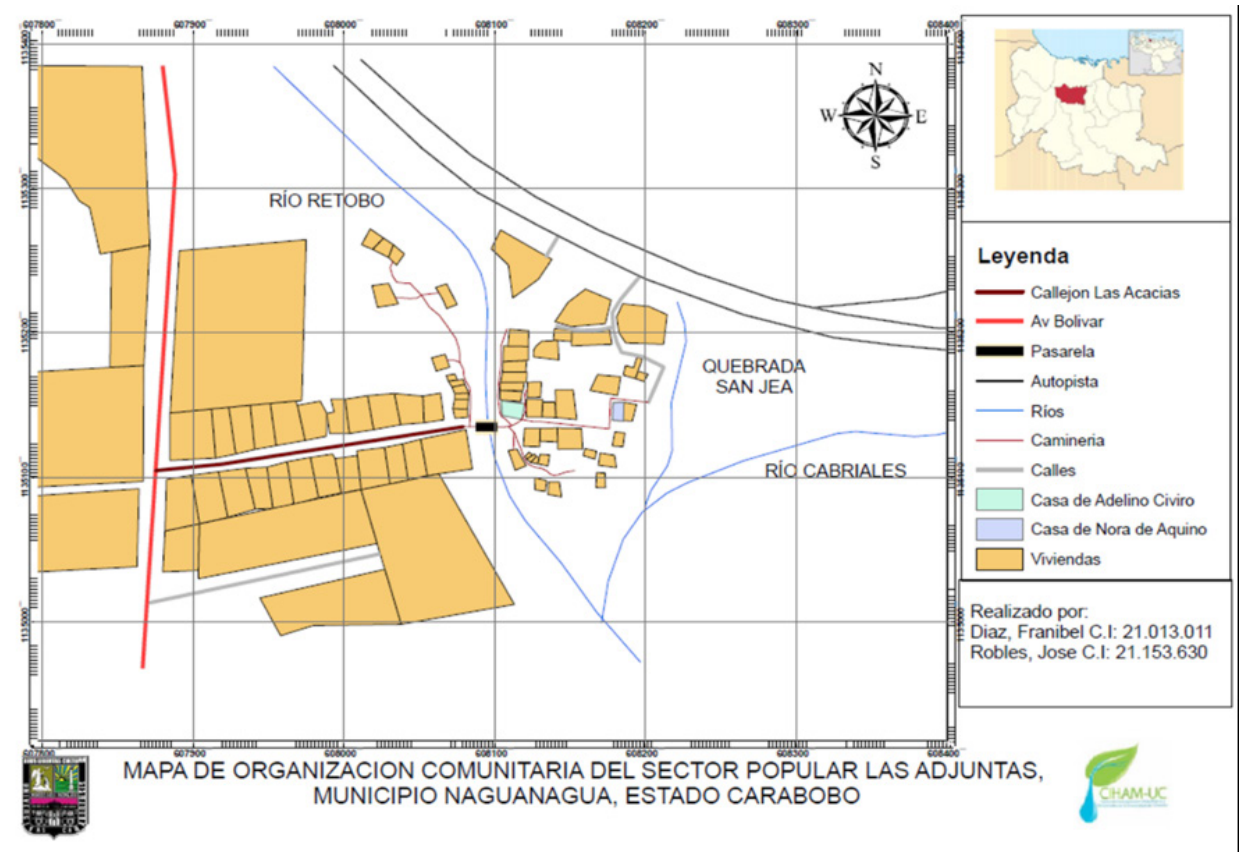

Figura 5. Mapa de organización comunitaria en la CPLA-MN Fuente: Autores, 2020.

En la Figura 6 se muestran las rutas de evacuación que se seleccionaron con participación de vecinos, personal de protección civil y bomberos.

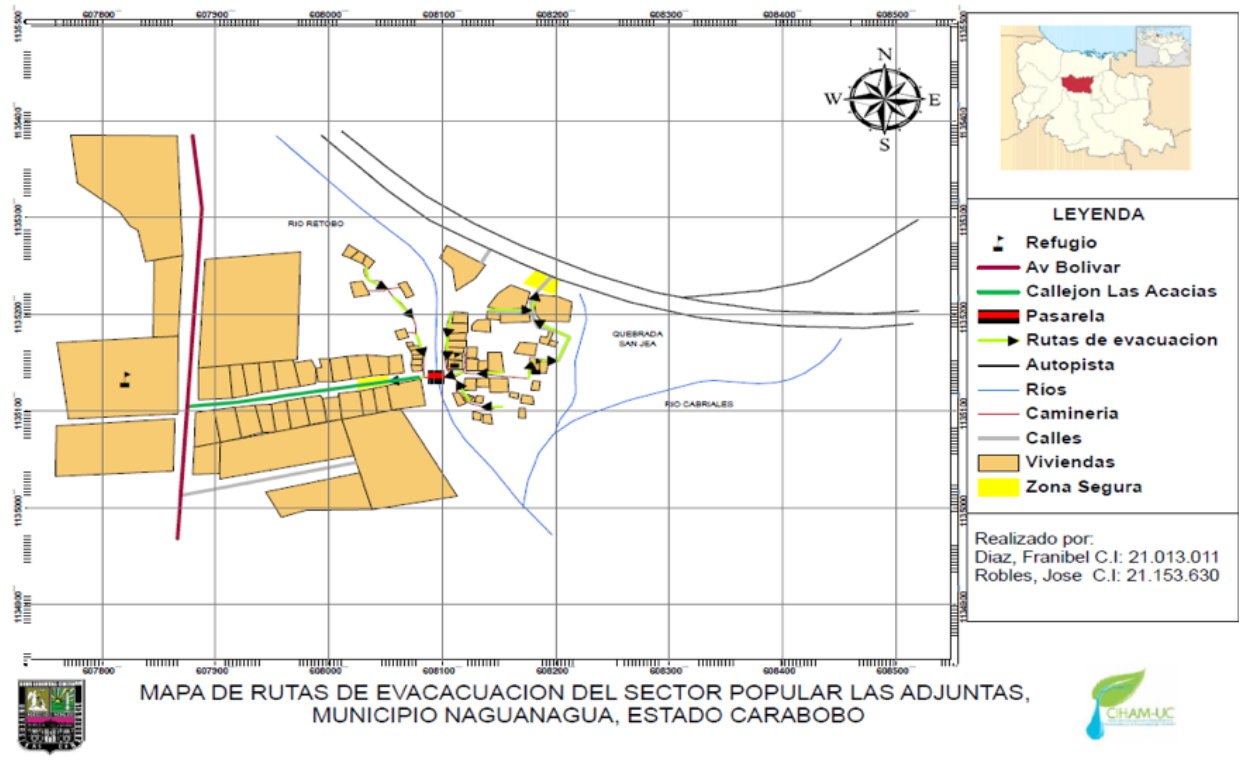

Figura 6. Rutas de evacuación en caso de inundación en la CPLA Fuente: Autores, 2020.

El criterio de selección de las zonas seguras ante la presencia de inundación fue la ubicación de las áreas con mayor cota sobre el terreno.

En la Figura 7 se indican los sitios más importantes para la CPLA, tales como la ubicación de puntos de asistencia médica, organismos de seguridad y rescate, farmacias, iglesias, alcaldía, entre otros. 

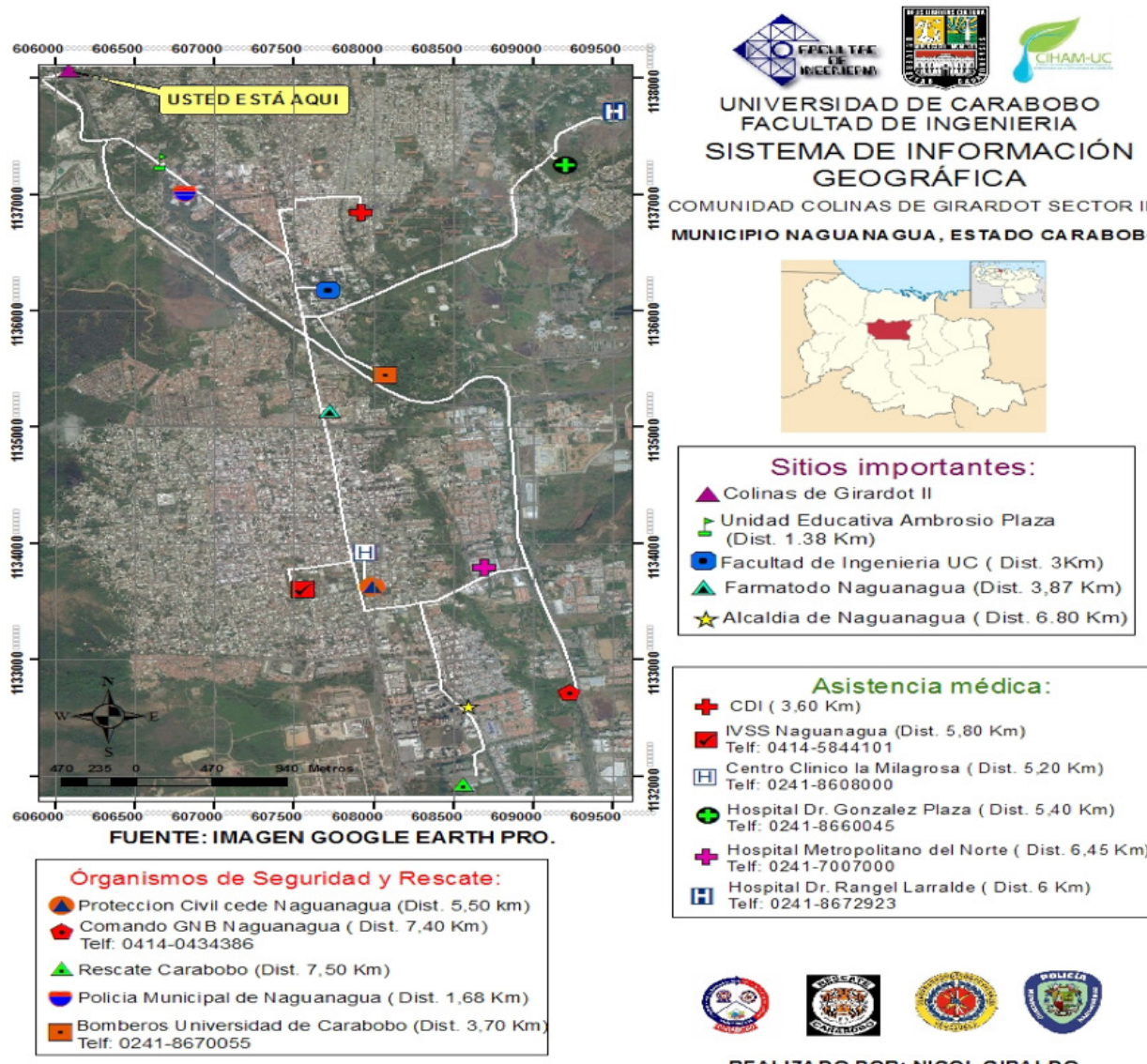

COMUNIDAD COLINAS DE GIRARDOT SECTOR ॥ MUNICIPIO NAGUANAGUA, ESTADO CARABOBO

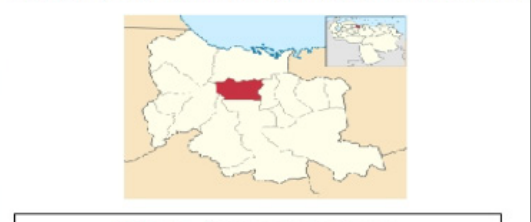

Sitios importantes: A Colinas de Girardot II

Unidad Educativa Ambrosio Plaza

- Facultad de Ingenieria UC ( Dist. $3 \mathrm{Km}$ ) $\Delta$ Farmatodo Naguanagua (Dist. $3,87 \mathrm{Km}$ ) ₹ Alcaldia de Naguanagua (Dist. $6.80 \mathrm{Km}$ )
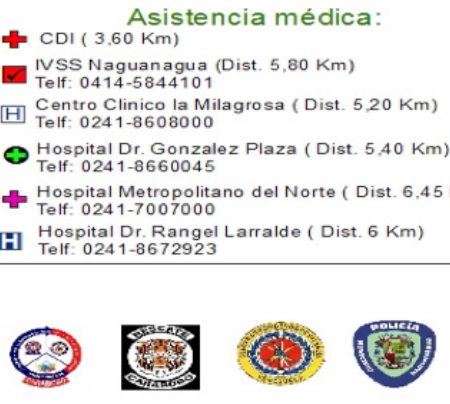

REALIZADO POR: NICOL GIRALDO

Figura 7. Ubicación de recursos locales en las comunidades del M. Naguanagua Fuente: Autores, 2020.

\section{Organización de acciones posibles de respuesta}

Se presentan resultados de las medidas estructurales y no estructurales aplicadas para enfrentar y afrontar las situaciones de riesgo.

\section{Medidas no estructurales:}

1) Implementación de planes de educación y divulgación sobre las acciones de gestión de riesgo.

Las escenas de la Figura 8 ( $A, B, C)$ muestran actividades de primeros auxilios, traslado y atención de heridos, simulacro de desalojo en una oficina pública, y charlas sobre temas relacionados con planes de gestión de riesgos, como parte del entrenamiento básico aplicado al personal de UC y del CIHAM-UC. La Figura 8 (D, E, F) muestra imágenes de los cursos, charlas y visitas puerta a puerta que se ofrecieron en la comunidad para iniciarlos en los temas de gestión de riesgos, así como levantamiento de información en forma conjunta con los miembros de la CLA-MN.
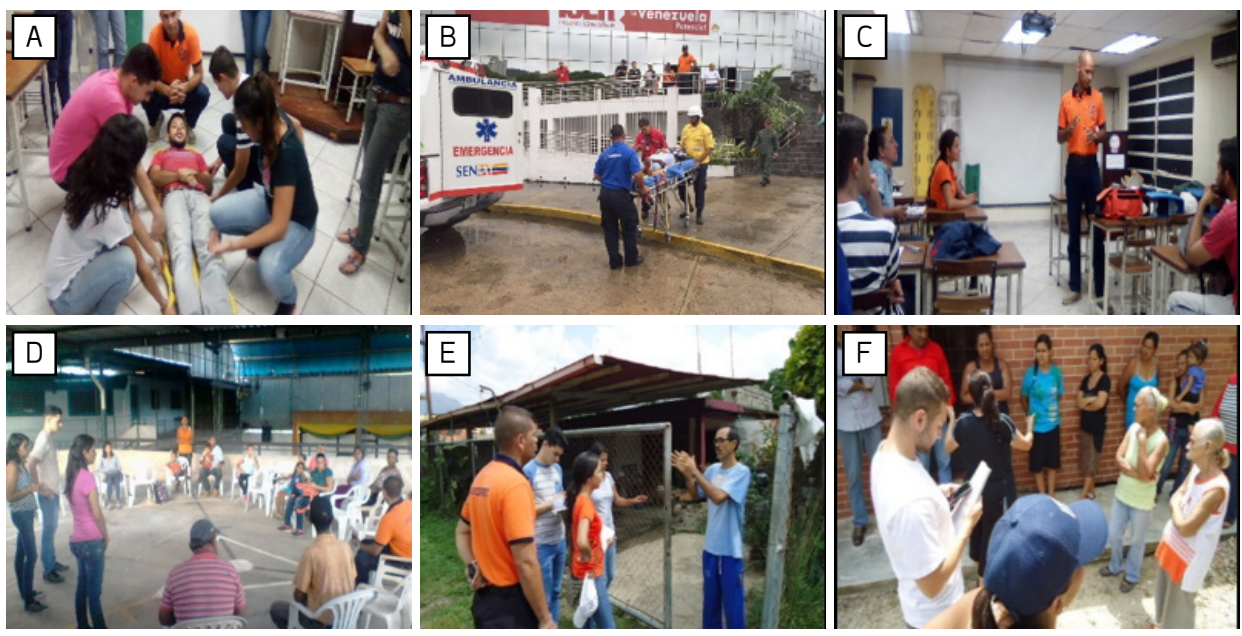

Figura 8. Escenas de algunas actividades realizadas en el curso dictado por Protección Civil: charlas, reuniones, visitas puerta a puerta realizadas a los miembros CPLA Fuente: Autores, 2020.

En la Figura 9 (A, B, C) se observan imágenes de la socialización de los productos elaborados 
en esta fase donde se presenta a la comunidad los mapas de ubicación de los sitios seguros, las vías de evacuación, y las viviendas más propensas a inundarse.
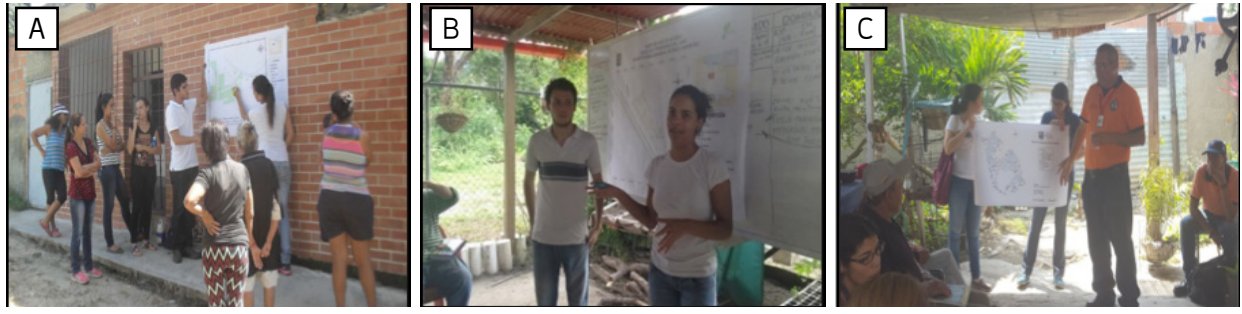

Figura 9. Escenas de algunas actividades realizadas durante la socialización de los productos elaborados durante la preparación sobre gestión de riesgo de inundación a los miembros CPLA Fuente: Autores, 2020

En la Figura $10(A, B, C)$ se observan imágenes de las charlas y los productos elaborados en esta fase como juegos didácticos y pluviómetros caseros a los estudiantes de la Comunidad Educativa Nuestra Señora de Fátima.
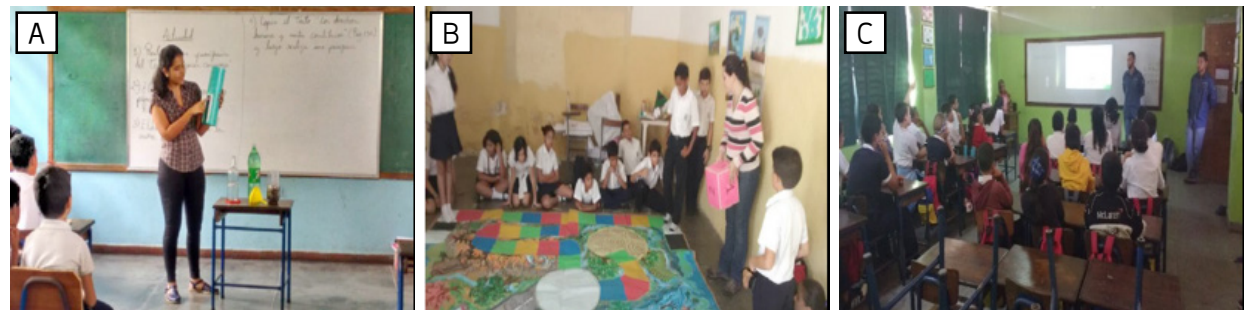

Figura 10. Escenas de algunas actividades correspondientes a socialización de productos con los estudiantes del colegio Nuestra Señora de Fátima ubicada en CPLA Fuente: Autores, 2020

\section{2) Sistema de Alerta Temprana (SAT)}

Se seleccionó un SAT integrado por pluviómetros comunitarios, linnímetro y sensores de nivel ubicados en el cauce, conectados a un tablero de control con una alarma sonora y una visual que indica a las personas que el nivel de agua va alcanzando los umbrales definidos de acuerdo a la experiencia de inundaciones anteriores de la comunidad, permitiéndoles desalojar sus casas y dirigirse con tiempo a los sitios de resguardo de acuerdo a los tiempos estimados por los simulacros realizados. La instalación de los instrumentos que conforman el SAT fue realizada por los miembros de la comunidad que apoyaron en todas las actividades programadas. En la Figura 11(A) se muestran escenas correspondientes a la instalación del linnímetro, siendo soldado a la pasarela y donde los habitantes pueden visualizar la altura que alcanza el agua del río -está marcado con colores de acuerdo a la escala de riesgos definida con los miembros de la comunidad. La Figura 11(B) presenta escenas de instalación del pluviómetro. La Figura 11(C) muestra la instalación del tablero del sistema de sensor de nivel. El monitoreo del SAT quedó en manos de los vecinos que están ubicados frente la pasarela donde se instalaron los equipos, y mediante mensajería de texto se avisa al resto de la comunidad.
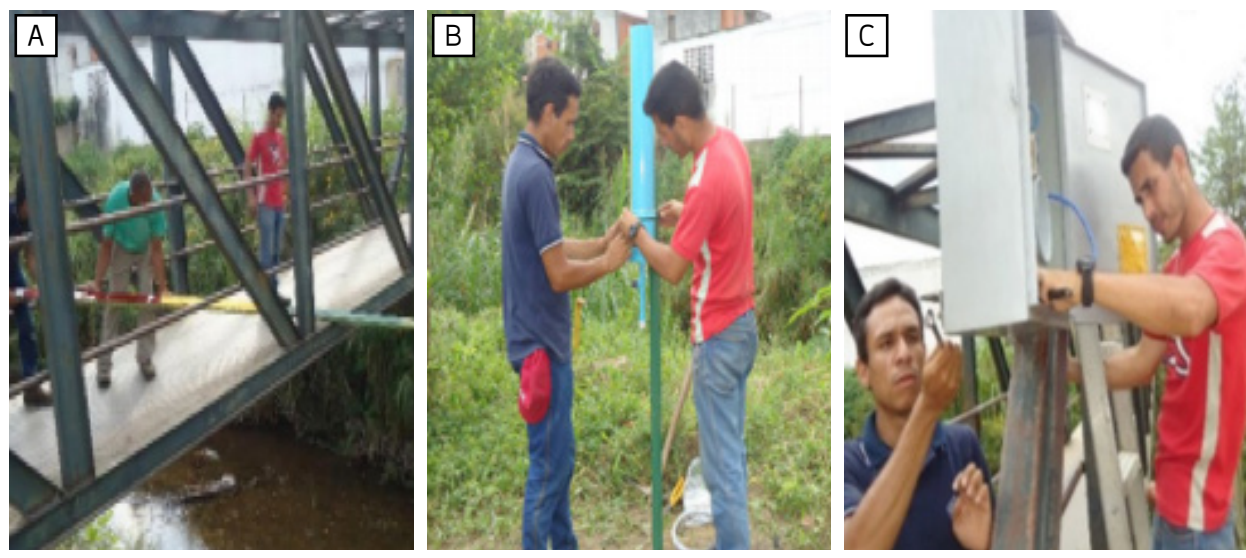

Figura 11. Instalación de SAT implementado en la CPLA-MN Fuente: Autores, 2020. 


\section{3) Mantenimiento del cauce de los ríos}

Como otra medida no estructural se limpiaron los cauces de los ríos con el apoyo de los concejales del municipio Naguanagua, aportando maquinaria pesada y camiones para bote de escombros y material removido del fondo del río. En la Figura $12(A, B, C)$ se muestran las escenas correspondientes a estas actividades.
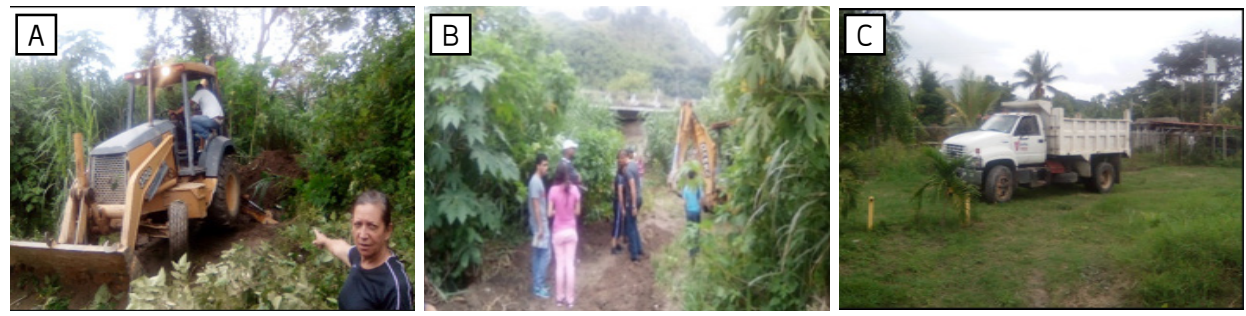

Figura 12. Limpieza del cauce como medidas no estructurales implementadas en la CPLA-MN Fuente: Autores, 2020

\section{Medidas estructurales:}

Luego de la simulación en el software HEC-RAS 4.0, se pudo observar que el flujo del tramo del rio El Retobo en confluencia con el rio Cabriales presenta un régimen gradualmente variado, con un comportamiento supercrítico predominante debido a la pendiente del cauce y la canalización de concreto existente, además se observó que en la progresiva $0+350$ aproximadamente debido a un cambio brusco de pendiente se forma resalto hidráulico que ocasiona que aguas abajo el nivel de agua incremente ocasionando que la comunidad popular de Las Adjuntas se inunde desde la progresiva 0+075 hasta la 0+225, ocasionando grandes daños. Al mismo tiempo se pudo determinar que la pasarela que conduce a la comunidad en la progresiva 0+140 está ocasionando un incremento en el nivel del fluido aguas abajo.

Los resultados obtenidos de la simulación fueron importantes para el planteamiento de las medidas estructurales en esta investigación, permitieron determinar los niveles y las planicies inundables, evidenciando que en los tramos del río próximos a la comunidad ocurre desbordamiento para periodo de retorno de 100 años.

Se proyectaron como medidas estructurales. 1) La protección de márgenes del río con muros de gaviones, ya que este tipo de estructuras presenta ventajas que se adaptan a las necesidades del proyecto, una de ellas su integración ecológica con el ambiente, además de ser una propuesta más económica, dado que para su construcción no amerita personal calificado, se pueden utilizar materiales existentes del sitio y reduciendo los costos de materiales y mano de obra. 2) Canalización de fondo móvil del río. Esta se prolongará desde la confluencia con el río Cabriales hasta la progresiva final de la canalización de concreto existente (300 metros aguas arriba desde la confluencia con el río Cabriales), procurando el encauzamiento del río y protegiendo así los márgenes. La canalización no aumentará la capacidad hidráulica del cauce, esta se adaptará a las condiciones geomorfológicas actuales.

En la Figura 13 se muestra un detalle de la canalización con muro de gaviones propuesta.

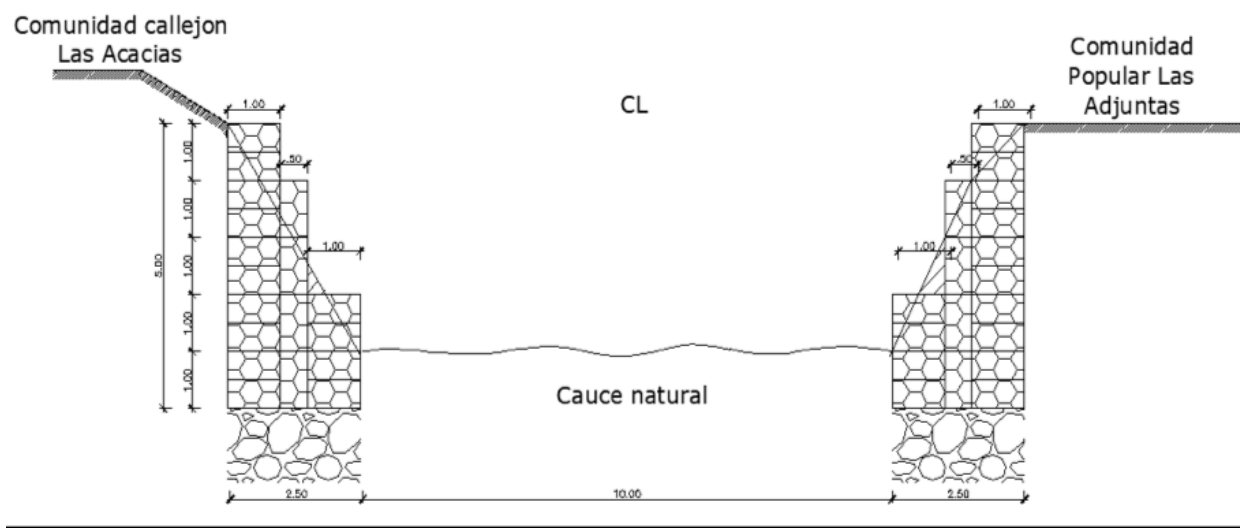

Figura 13. Detalle de canalización con muro de gaviones diseñado

Fuente: Autores, 2020. 


\section{VALIDACIÓN Y SOSTENIBILIDAD DEL PGSRI-MN}

Se presenta en esta fase los resultados de la validación y monitoreo del plan donde se aplicaron encuestas a los líderes y miembros de la comunidad, al personal docente y administrativo del colegio Nuestra Señora de Fátima, bomberos de la UC, personal de protección civil, personal de la Universidad de Carabobo, y miembros de la alcaldía del municipio. En la comunidad se entrevistaron 150 personas y la totalidad de las personas encuestadas respondieron que se sienten beneficiados con este PGSRI-MN. En los colegios se seleccionó una muestra de 80 personas entre alumnos, docentes, y representantes, el 100\% de las personas entrevistadas está de acuerdo que es un beneficio para los alumnos conocer sobre el PGSRI, en cuanto al personal de Protección Civil y los Bomberos manifestaron su satisfacción por el trabajo en conjunto realizado, para ellos es un trabajo que merece apoyo y ser replicado en otros municipios. La Figura 14(A) muestra el resultado de la pregunta propuesta antes de la aplicación PGSRI-MN: ¿Cree Usted que la aplicación de un PGSRI-MN puede ser una medida efectiva para ayudar a esta comunidad? El $67 \%$ de los entrevistados respondía no saber si el PGSRI-MN podría funcionar en la comunidad una vez desarrollado e implementado el PGSRI-MN se realizó la misma pregunta. La Figura 14(B) muestra el resultado a esta misma pregunta donde el $100 \%$ de los involucrados en el proyecto aseguran que el PGSRI es una medida efectiva y útil para su comunidad.
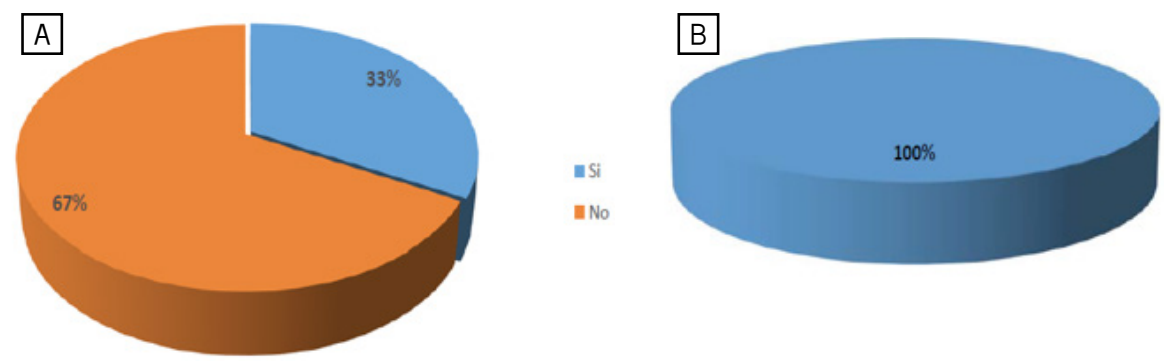

Figura 14. Resultados de la aplicación de la encuesta de la validación del PGSRI-MN Fuente: Autores, 2020.

Según el Informe Brundtland Comisión Mundial sobre Medioambiente y Desarrollo (WCED, 1987), el modelo de desarrollo sostenible debe ser la combinación de un grado de desarrollo económico y de calidad de vida que no comprometa la capacidad del medioambiente para sustentar a la población en el futuro, proporcionando un grado de bienestar económico a las generaciones actuales y venideras a la vez que se mantiene el buen estado del medioambiente. Desde este punto de vista, la implementación del PSGRI en el Municipio Naguanagua y el trabajo de esta investigación constituyen un aporte a la sostenibilidad de la gestión de riesgos de inundación, ya que la gestión del riesgo de desastre consiste en la capacidad para evitar que las amenazas se cumplan y pasen a ser desastres. Los criterios técnicos y operativos dedicados a la identificación de las amenazas en esta investigación abren la posibilidad para la consolidación de un desarrollo sostenible que implica la reducción de vulnerabilidades y el mejoramiento de la calidad de vida como parte del proceso social, convirtiéndose en un elemento fundamental para la construcción de territorios seguros que aporten al desarrollo territorial sostenible.

La participación ciudadana juega un papel muy importante en la sostenibilidad de este tipo de proyectos ya que la baja o nula participación, en términos de asociatividad, aumentan los niveles de riesgo impidiendo respuestas rápidas y efectivas ante situaciones adversas. En este proyecto los habitantes fueron receptivos a la información suministrada en todo el entrenamiento, y por los resultados obtenidos en la encuesta de validación, se evidenció la disposición a continuar monitoreando el SAT instalado y transmitir dicha información a sus vecinos y familiares. Aunque los resultados son una base para evaluar, no garantizan que esta se mantenga en el tiempo, por lo que se diseñó un plan de charlas periódicas para reforzar los conocimientos de la comunidad y que estos perduren en el tiempo, las cuales serán dictadas por los servicios municipales de protección civil y los bomberos.

Por su parte, tanto la Universidad de Carabobo como el CIHAM-UC se comprometen a seguir financiando investigaciones de este tipo y se plantean nuevos retos: 1) Actualización del PGSRI-MN para el periodo 2019-2020; 2) Implementar SAT automatizados mediante tecnología de punta con el uso del software Arduino; 3) Ampliar la cobertura del programa de los Planes Escolares para la Gestión de Riesgos; 4) Desarrollar programas para la socialización de la prevención y la mitigación de desastres. 


\section{CONCLUSIONES}

La formulación del PGSRI-MN fue una propuesta desde la Universidad de Carabobo a las comunidades del Municipio Naguanagua, orientada a promover el conocimiento y la preparación para la prevención y mitigación, mediante la promoción de actividades participativas e interinstitucionales. En nuestra experiencia, estas comunidades cuentan con principios de convivencia social y con actitud crítica orientada hacia la reflexión y la organización comunitaria que permitieron apoyar y poner en práctica planes de gestión de riesgos que les permitirán salvar vidas en el futuro. Los proyectos correspondientes a las medidas estructurales fueron entregados a los líderes comunitarios para que se soliciten los recursos a los entes encargados dar soluciones a estos casos. En cuanto a las medidas no estructurales, éstas se ejecutaron con el aporte económico de CIHAM-UC, los estudiantes, y la Alcaldía de Naguanagua. Los vecinos aportaron la mano de obra en su totalidad y continúan monitoreando el SAT con el apoyo de protección civil. Por su parte, los bomberos universitarios continúan su preparación en gestión de riesgos con la comunidad.

En conversaciones con los vecinos, pudimos constatar que esta experiencia ha sido enriquecedora para la comunidad porque les permitió integrarse con los estudiantes, pudiendo compartir experiencias, logrando un aprendizaje conjunto y un respeto y reconocimiento mutuo. Por otra parte, para los docentes de la UC esta experiencia también fue beneficiosa ya que pudieron desarrollar su pensamiento crítico y creativo, en el desarrollo de estrategias adaptadas a la realidad actual de la sociedad la cual exige más participación y difusión de los conocimientos adquiridos. Otro grupo beneficiado fueron los estudiantes, quienes conocieron de primera mano las necesidades de sus comunidades, y experimentaron como pueden llegar a liderar proyectos comunitarios en beneficio de los más desfavorecidos, alcanzando a la vez una formación integral como ingenieros e ingenieras civiles.

\section{REFERENCIAS}

Farias, B., Márquez, A., Guevara, E., \& Rey, D. (2018). Characterization spatio-temporal land use in watershed using geomatic techniques. Revista Ingeniería UC, 25(1), 19-30. Recuperado de: http:// servicio.bc.uc.edu.ve/ingenieria/revista/v25n1/vol25n12018.pdf [visitado el 1o de nov. 2019]

Farías, B., Márquez A., Guevara E. \& Romero, A. (2017). Una metodología para prevenir y afrontar el riesgo hidrológico en la zona norte del municipio Naguanagua estado Carabobo - Venezuela. Revista Encuentros Unellez, 1(1), 86-102.

Farías, B., Márquez, A. \& Guevara, E. (2015, noviembre). Evaluación del grado de sostenibilidad de la aplicación de simulacros de gestión de riesgos desastres en el Estado Carabobo. Aceptado para el IV Congreso Nacional de Ciencia y Tecnología, Caracas, Venezuela, o4-o6 Nov.

Guevara, E. (2010). Gerencia de desastres. Valencia, Venezuela: Asociacion de Profesores UC, Universidad de Carabobo.

Guevara, E. (2008). Educación para confrontar desastres. Valencia, Venezuela: Vicerrectorado Académico, Dirección General de Postgrado, Universidad de Carabobo.

Guevara, E. (2003). El hombre y su ambiente. Valencia, Venezuela: Dirección General de Medios, Universidad de Carabobo.

Organización de los Estados Americanos OEA. (1993). Manual Sobre Manejo de Peligros Naturales en la Planificación para el Desarrollo Regional Integrado. Washington, D.C.: OEA.

Organización Meteorológica Mundial OMM. (2017). Aspectos destacados de 2016. Informe Anual. Ginebra: OMM.

Organización Meteorológica Mundial OMM. (2006). Aspectos Ambientales de la gestión integrada de crecidas. APFM Documento Técnico, 3. Serie Políticas de Gestión de Crecidas. Ginebra: OMM.

Organización de las Naciones Unidas para la Educación, la Ciencia y la Cultura UNESCO. (2012). Proyecto Regional DIPECHO VII UNESCO-CEPREDENAC. Gestión para la Reducción del Riesgo de Desastres y Sistemas de Alerta Temprana. Manual para Docentes. San José, Costa Rica: UNESCO. Recuperado de: http://www.unesco.org/new/es/sanjose/natural-sciences/proyecto-dipecho/ [visitado el 10 de nov. 2019]

United Nations International Strategy for Disaster Reduction UNISDR. (2015). Sendai Framework for Disaster Risk Reduction 2015-2030. Sendai: UNISDR.

Vásquez, R., Manrique, I. \& Ramos, R. (2014, junio). SIG aplicado a la evaluación de vulnerabilidad por inundación en la ciudad de Tixtla, Guerrero. México. Aceptado para el XVI Congreso Nacional de Tecnologías de la Información Geográfica, Alicante, España.

World Commission on Environment and Development WCED. (1987). Our common future. Oxford: Oxford University Press. 